\title{
Late-Onset Seizures Associated with Quetiapine Poisoning
}

\author{
Amy C. Young, MD, Kurt C. Kleinschmidt, MD, Paul M. Wax, MD
}

Division of Emergency Medicine, UT Southwestern Medical Center, Dallas, TX

\begin{abstract}
Introduction: Quetiapine, a second-generation antipsychotic, acts at multiple brain neurotransmitter receptors and has the potential for serious complications. Although seizures have been described in the literature, delayed seizure onset has not been reported. We report the first case of delayed seizures after a significant quetiapine overdose.

Case Report: A 27-year-old female presented to the emergency department following an overdose of approximately $30 \mathrm{~g}$ of quetiapine. Twenty-four hours after arrival, the patient had 2 seizures. The patient was then intubated and remained in the ICU for four days. EEG was negative for epileptiform activity. The serum quetiapine levels (MedTox, St. Paul, MN) were 8.67 mg/L on hospital day one and $3.28 \mathrm{mg} / \mathrm{L}$ on hospital day three.

Discussion: Quetiapine poisoning, with serum levels, associated with seizures has been reported in one prior case. Our case report represents late-onset seizures with serum levels above therapeutic range $(>1 \mathrm{mg} / \mathrm{L})$. The serum concentrations of quetiapine in this case were consistent with those in postmortem case reports.
\end{abstract}

\section{INTRODUCTION}

Quetiapine in overdose can have serious consequences, although fatalities are rare. It is most commonly associated with sinus tachycardia, prolonged QTc intervals, and central nervous system depression [1]. Patients may require intubation for respiratory depression with prolonged ICU stays for larger overdoses [1]. Other less common effects include seizures and hypotension [2]. Seizures starting 24 hours after overdose have not been reported with quetiapine overdoses. This case reflects the potential for these complications.

\section{CASE REPORT}

A 27-year-old female presented to the emergency department (ED) following an ingestion of approximately $30 \mathrm{~g}$ of quetiapine (usual therapeutic dose is $150-800 \mathrm{mg}$ per day). The patient became more sedated en route and was somnolent on ED arrival. No further history was obtained.

In the $\mathrm{ED}$, the patient was unresponsive. Vital signs were: blood pressure, $110 / 54 \mathrm{mmHg}$; pulse, 132/min; respirations, 26/min; temperature, $36.9^{\circ} \mathrm{C}$; and room air pulse oximetry, $93 \%$ saturation. There was no seizure activity during prehospital transport, ED stay, or the initial 24 hours in the hospital. The patient was admitted for observation and remained unresponsive, but developed moderate respiratory distress at 24 hours. An arterial blood gas on $4 \mathrm{~L}$ nasal cannula showed a $\mathrm{pH}$ of $7.38, \mathrm{paCO}_{2}$ of $35 \mathrm{mmHg}, \mathrm{paO}_{2}$ of $51 \mathrm{mmHg}$, and $\mathrm{SaO}_{2}$ of $84 \%$. The patient was transferred to the ICU and vital signs were: blood pressure, 84/42 mmHg; pulse, 136/min; respirations, 20/min; temperature, $38.3^{\circ} \mathrm{C}$; and non-rebreather pulse oximetry, $94 \%$ saturation. The physical findings included equal but slowly reactive 3-mm pupils, dry mucous membranes, and chest exam with diffuse rales greater on the right than the left, no abnormal cardiac sounds, a normal abdominal exam, and extremities without edema. Neurologically, the patient localized, mumbled, and opened eyes to pain, which was unchanged from ED arrival. Reflexes were normal. Intravenous fluids of 1-L normal saline were given for hypotension after ICU arrival. The patient then had a 1-minute, generalized tonic-clonic seizure that resolved spontaneously. The patient remained sedated following the seizure activity. During a CT scan, the patient had another witnessed tonic-clonic seizure about 1 hour after the

Keywords: quetiapine, overdose, seizure, serum level

Notes: There was no outside funding of any kind used for this study.

Corresponding Author: Amy C. Young, MD, 5323 Harry Hines, Dallas, TX 75390. Email: acyoung_2000@yahoo.com 
initial seizure activity, which lasted $<1$ minute. She was then intubated. The head CT was normal.

The patient was given lorazepam and diprovan, and no further seizures occurred. An electrocardiogram (ECG) showed sinus tachycardia at 125 beats/minute, a QTc of $409 \mathrm{~ms}$, and a QRS of $100 \mathrm{~ms}$. Blood tests at this time revealed: WBC of $22,100 / \mathrm{mm}^{3}$ with $93 \%$ polys, a hematocrit of $41 \%$, and 275,000 platelets $/ \mathrm{mm}^{3}$, sodium, $141 \mathrm{mEq} / \mathrm{L}$; potassium, $4.2 \mathrm{mEq} / \mathrm{L}$; chloride, $109 \mathrm{mEq} / \mathrm{L}$; bicarbonate, $21 \mathrm{mEq} / \mathrm{L}$; blood urea nitrogen, $10 \mathrm{mg} / \mathrm{dL}$; creatinine, $0.99 \mathrm{mg} / \mathrm{dL}$; and glucose, $87 \mathrm{mg} / \mathrm{dL}$. The lactate was $7.7 \mathrm{mmol} / \mathrm{L}(0.7-2.1 \mathrm{mmol} / \mathrm{L})$. The urine pregnancy test and the urinalysis were negative. Acetaminophen, aspirin, and ethanol levels were all negative. The urine drug screen immunoassay (AxSYM, Abbott Laboratories, Abbott Park, IL) was negative for cannabinoids, amphetamines, PCP, benzodiazepines, barbiturates, cocaine, and opiates. Cerebrospinal fluid was normal. The creatinine kinase (CK) value after the seizure was 860 units/L (40-210 units/L). AST and ALT were normal. Chest radiograph following intubation showed a right pleural effusion with mild pulmonary edema. The patient was started on ticarcillin and clavulanate for possible aspiration pneumonia.

The patient had an EEG that demonstrated no epileptiform activity. There was no further seizure activity during her hospital stay. The serum quetiapine levels (MedTox, St. Paul, MN) were $8.67 \mathrm{mg} / \mathrm{L}$ on hospital day 1 and $3.28 \mathrm{mg} / \mathrm{L}$ on hospital day 3 . Therapeutic levels have been reported as 0.1 to $1.0 \mathrm{mg} / \mathrm{L}$ [3]. On hospital day 4 , the patient was more alert, followed commands, and was extubated. Daily ECGs revealed sinus tachycardia with normal intervals. She was discharged to a psychiatric center 6 days after arrival.

\section{DISCUSSION}

Quetiapine fumarate (Seroquel) is an atypical antipsychotic that antagonizes cerebral serotonergic 5-HT1A, 5-HT2, dopaminergic D1 and D2, histaminic H1, muscarinic M1, and adrenergic $\alpha-1$ and $\alpha-2$ receptors $[3,4]$. It is rapidly absorbed orally, attains peak plasma concentration in 1-2 hours, is metabolized by liver cytochrome p450 3A4 isoenzyme to the active metabolite 7 hydroxyquetiapine, and is excreted primarily in the urine $[1,4,5]$.

Most typical antipsychotics (e.g., phenothiazines) lower the seizure threshold more than atypical antipsychotics. Of the latter group, clozapine is the most frequently associated with seizures [6]. Quetiapine has a similar structure to the prototype, clozapine [4]. During the clinical trials for quetiapine, seizures occurred in $18(0.6 \%)$ of 2792 treated patients compared with $1(0.2 \%)$ of 607 on placebo [7]. In a case series of 18 patients with quetiapine overdose, 2 had seizures, with only 1 patient having a reported time of seizure onset [1].

The risk for having an EEG abnormality varies widely among antipsychotics: higher with clozapine and olanzapine, moderate with risperidone and typical neuroleptics, and lower with quetiapine [8]. A series of 22 patients taking a therapeutic dose of quetiapine demonstrated EEG abnormalities, but none of the patients had epileptiform activity. EEG abnormalities correlated with dose in the olanzapine group in contrast to patients treated with haloperidol, quetiapine, or healthy subjects [9]. Quetiapine poisoning in our case did not have EEG abnormalities.

Several case studies have described the clinical effects and tissue distributions of quetiapine in overdose. A case series, described by Balit et al., of 45 quetiapine overdoses with a median dose of $3.5 \mathrm{~g}$ had a median peak drug concentration of $2.59 \mathrm{mg} / \mathrm{L}$ [1]. Seizures occurred in 2 patients and mechanical ventilation was used in 4 patients. For 1 seizure patient, neither the timing of the seizure nor quetiapine levels was noted. The other patient had a 15- to 20-second tonic-clonic seizure starting 4 hours after ingestion. The peak level in this case was $20.48 \mathrm{mg} / \mathrm{L}$ [1]. Another case, without a serum quetiapine level, reported seizure-like activity on presentation to the ED after $4.5 \mathrm{~g}$ of quetiapine. The EEG did not show epileptiform activity [6]. These published reports suggest that seizures occur early after ingestion (at 4 hours or upon presentation), unlike our patient who had late-onset seizure activity commencing 24 hours after ingestion.

The reported therapeutic range of quetiapine is 0.1 to $1.0 \mathrm{mg} / \mathrm{L}$ $[3,4]$. However, plasma quetiapine levels and clinical response do not correlate. Thus, the monitoring of levels in clinical practice is controversial [1].

A 20-case review suggests that postmortem blood quetiapine concentrations $>1 \mathrm{mg} / \mathrm{L}$ may be associated with death [4]. Hopenwasser et al. reviewed 8 medical examiner cases. The article suggests that postmortem therapeutic concentrations that were not related to overdose occur with blood levels between 0.15 and $2.7 \mathrm{mg} / \mathrm{L}$ [5]. Drugs with increased lipophilicity, low protein binding, and increased diffusion across gastrointestinal tract into tissues as well as drugs with volumes of distribution $>3 \mathrm{~L} / \mathrm{kg}$ may contribute to increased postmortem redistribution [11]. According to Parker and McIntyre, the volume of distribution of $8-10 \mathrm{~L} / \mathrm{kg}$ indicates variability of quetiapine concentration among specimen types, and overall mean data suggests that its postmortem redistribution is small [12].

This case report has several limitations. Although the patient stated she had a single drug ingestion, there could be undetected coingestants. The patient stated that her only medication was quetiapine; however, she could be on other medications that could lower her seizure threshold. These limitations apply to most toxicology case reports. The patient could be withdrawing from a medication that may have resulted in a negative urine drug screen. However, she manifested no signs of a sedative-hypnotic withdrawal syndrome except tachycardia, a common symptom with quetiapine overdose. In addition, the patient experienced hypoxia, which could have resulted in late-onset seizures. However, the first seizure occurred 4 hours after her respiratory compromise had been recognized and treated with supplemental oxygen.

Although seizures have been reported with quetiapine poisoning, the serum levels at which these seizures occur have been reported in only one other case. There are many instances in which a "lethal" level in one person would be a "nonlethal" level in another person. Our case reports a very high level that has 
been associated with fatalities in previous studies. We report a case of initial and recurrent seizure onset 24 hours after ingestion of quetiapine.

The authors have no potential financial conflicts of interest to report.

\section{REFERENCES}

1. Balit CR, Isbister GK, Hackett PL, Whyte IM. Quetiapine poisoning: a case series. Ann of Emerg Med 2003;42:751-758.

2. Strachan PM, Benoff BA. Mental status change, myoclonus, electrocardiographic changes, and acute respiratory distress syndrome induced by quetiapine overdose. Pharmacotherapy 2006;26(4):578-582.

3. Langman LL, Kaliciak HA, Carlyle S. Case report: fatal overdoses associated with quetiapine. J Anal Toxicol 2004;28.

4. Flammia DD, Valouch T, Venuti S. Case report: tissue distribution of quetiapine in 20 cases in Virginia. J Anal Toxicol 2006;30:287-292.

5. Hopenwasser J, Mozayani A, Danielson T, et al. Postmortem distribution of the novel antipsychotic drug quetiapine. J Anal Toxicol 2004;28:264-268.
6. Hedges D, Jeppson K, Whitehead P. Antipsychotic medication and seizures: a review. Drugs Today (Barc) 2003;39: 551-557.

7. Quetiapine (Seroquel®) Package Insert. AstraZeneca Pharmaceuticals, October 2006.

8. Centorrino F, Price BH, Tuttle M, et al. EEG abnormalities during treatment with typical and atypical antipsychotics. Am J Psychiatry 2002;159:109-115.

9. Amann BL, Pogarell O, Mergl R, et al. EEG abnormalities associated with antipsychotics: a comparison of quetiapine, olanzapine, haloperidol and healthy subjects. Hum Psychopharmacol 2003;18:641-646.

10. Mauri MC, Volonteri LS, Colasanti A, et al. Clinical pharmacokinetics of atypical antipsychotics: a critical review of the relationship between plasma concentrations and clinical response. Clin Pharmacokinet 2007;46: 359-388.

11. Rodda KE, Drummer OH. The redistribution of selected psychiatric drugs in post-mortem cases. Forensic Sci Int 2006; 164:235-239.

12. Parker DR, McIntyre IM. Case studies of postmortem quetiapine: therapeutic or toxic concentrations? J Anal Toxicol 2005;29:407-412. 